\title{
Handling relapse in smoking cessation: strategies and recommendations
}

\author{
Pasquale Caponnetto $\cdot$ Elaine Keller • \\ Cosimo M. Bruno $\cdot$ Riccardo Polosa
}

Received: 12 April 2012/ Accepted: 22 September 2012/Published online: 7 October 2012

(C) SIMI 2012

\begin{abstract}
Once established, smoking is a very difficult addiction to break. Many smokers persist in tobacco use for several years and typically cycle through multiple periods of remission and relapse. Smoking cessation is not a single event but a process, and relapse is an ordinary component of this process. While international guidelines place great emphasis on relapse prevention, very little can be found about managing smokers who have relapsed. This article is intended to address the challenge of managing smokers who relapse in the course of a smoking cessation program. This knowledge may lead to an improved smoking cessation outcomes.
\end{abstract}

Keywords Smoking $\cdot$ Nicotine addiction $\cdot$ Smoking cessation $\cdot$ Relapse $\cdot$ Tobacco harm reduction

P. Caponnetto $\cdot$ R. Polosa

Centro per la Prevenzione e Cura del Tabagismo (CPCT), Azienda Ospedaliero-Universitaria "Policlinico-Vittorio

Emanuele”, Università di Catania, Catania, Italy

P. Caponnetto - C. M. Bruno · R. Polosa

Institute of Internal Medicine, G. Rodolico Hospital, Azienda Ospedaliero-Universitaria "Policlinico-Vittorio Emanuele",

Università di Catania, Catania, Italy

E. Keller

The Consumer Advocates for Smoke Free Alternatives

Association, Springfield, VA, USA

R. Polosa $(\square)$

Presidio G. Rodolico, Unità Operativa di Medicina

Interna e Medicina d'Urgenza, University of Catania,

Via S. Sofia 78, 95123 Catania, Italy

e-mail: polosa@unict.it

\section{Introduction}

Cigarette smoke harms nearly every system of the human body, thus causing a broad range of diseases, many of which are fatal. The risk of serious disease diminishes rapidly after quitting, and permanent abstinence is known to reduce the risk of lung cancer, heart disease, chronic lung disease, stroke, and other cancers [1].

Although evidence-based recommendations indicate that smoking cessation programs are useful in helping smokers to quit [2], the powerful addictive qualities of nicotine and of the ritualistic behavior of smoking create a huge hurdle, even for those with a strong desire to quit. Once established, smoking is a very difficult addiction to break. Many quitting smokers persist in tobacco use for several years, and typically cycle through multiple periods of remission and relapse. It has been shown that approximately $80 \%$ of smokers who attempt to quit on their own relapse within the first month of abstinence, and only about 3-5\% remain abstinent at 6 months [3]. This is not surprising given that tobacco dependence shows many features of a chronic disease, with the majority of smokers typically experiencing multiple periods of remission and relapse.

Therefore, smoking cessation is not a single event but a process, and relapse is an ordinary component of this process. Recognizing the relapsing nature of the condition underscores the importance for ongoing, rather than just acute, care. Several treatment options are now available to assist with repeated quitting attempts, and to decrease episodes of relapse [2, 4]. But, although international guidelines place great emphasis on relapse prevention [2], very little can be found about managing smokers who have relapsed. This is surprising considering that the typical patient undergoing smoking cessation will eventually relapse. 
In this article, we will draw attention to the challenge of assisting smokers who relapse in the course of their smoking cessation program, and provide practical advice for their management. This knowledge may lead to improved smoking cessation rates.

\section{Nicotine withdrawal drives relapse}

Upon inhalation of cigarette smoke, nicotine enters the blood stream, crosses the blood-brain barrier and binds to nicotinic receptors in the midbrain thereby increasing the release of neurotransmitters such as dopamine, which is responsible for the rewarding and addictive effects of nicotine. With long-term exposure, nicotine can increase the number of nicotinic receptors, and can cause some receptors to become desensitized. These changes in the brain structure and function may result in cessation-associated withdrawal symptoms that are known to be responsible for the impaired ability to achieve and sustain abstinence [5]. Symptoms that are valid indicators of nicotine abstinence include dysphoric or depressed mood, insomnia, irritability, anxiety, difficulty concentrating, restlessness, and cigarette craving [2, 6, 7].

These symptoms not only can cause clinically, cognitive, and social distress, but also are the main cause for relapse. Treatment of dependence and withdrawal can restore brain function, mood, and cognitive abilities, and thereby support cessation, but individuals appear to vary widely in how long they may require treatment, and probably in what forms of treatment are acceptable and effective.

A number of questionnaires have been developed to assess the presence and intensity of withdrawal symptoms. These include the Minnesota Nicotine Withdrawal Scale (MNWS), the Mood and Physical Symptoms Scale (MPSS), the Shiffman Scale (SS), the Wisconsin Smoking Withdrawal Scale (WSWS), and the Cigarette Withdrawal Scale (CWS) [8]. Administering one of these questionnaires before the quitting attempt will help identify the patients who need help in getting ready for such an attempt, and administering the questionnaire again on subsequent visits will help track the intensity and duration of the withdrawal symptoms. This may be important to adjust the treatment intensity and prevent relapse.

\section{Notes on relapse prevention}

Unpleasant surprises can undermine an attempt to quit smoking. Patients need to be equipped with knowledge of what to expect. Schnoll et al. [9] evaluated perceived control over abstinence-induced symptoms, and find that a greater sense of control predicts a higher rate of smoking cessation success. Informing patients about the possible withdrawal symptoms can help patients to normalize their experience. But, it is important to point out that some people do not experience any of these problems, and a few might experience all of them $[10,11]$. It also helps patients to be informed that the duration of these problems varies from person-to-person [3]. For most people, any withdrawal symptoms peak in the first few days and subside within 1-3 weeks [12]. For some people, the symptoms can last longer [12]. The patient should be urged to seek help if any problems arise that might cause him or her to relapse, and the health provider should be prepared to provide advice and medical assistance while dealing with these symptoms as soon as they emerge [13].

Numerous studies have been conducted to identify treatments that can reduce the likelihood of future relapse [14-16]. Many studies attempt to reduce relapse either by including behavioral support or by pharmacotherapy in the cessation treatment. With the exception of varenicline, which has been shown to offer significant improvement in abstinence rates when evaluated for long-term maintenance treatment [17], in general, such studies have shown that either behavioral or medication treatments are not very effective at reducing the likelihood of relapse [18]. At present, the best strategy for preventing relapse and producing high long-term abstinence rates appears to be the combined use of the most effective cessation medication available together with relatively intense cessation counseling [2]. However, in a not-too-distant future, it is possible that new approaches, such as nicotine vaccination, might prove beneficial for relapse prevention [19, 20].

Several predictors of success in smoking cessation can be used to identify probable relapsers, including late age of initiation of cigarette smoking, longer duration of previous quit attempts, lack of depression, low-to-moderate nicotine dependence, absence of alcohol problems, sustained level of motivation, being married, and not having any other smokers in the household [21]. Identification of individual characteristics that predict success in smoking cessation is highly desirable as this could help to match smokers with a strategy that is more likely to help them quit, to identify smokers who might need more intensive treatment, and to make the most of health care resources. For example, the notion that depressive symptoms significantly lower the smoking cessation, success rates [19, 22] account for the positive effect on quitting success of the antidepressant bupropion. Therefore, it could be helpful to advise patients who exhibit pre-treatment symptoms of depression that taking antidepressant medication will increase their odds of success. However, several smokers may resist treatment with antidepressant medication to avoid the stigma of being labeled as "mentally ill" [23]. Those who initially refuse 
should be counseled that depression is caused by a chemical imbalance, and is a treatable medical problem, not a character flaw.

\section{What to do in case of relapse? A practical algorithm}

Most smokers attempting to quit smoking experience episodes of relapse on the way to success. Nonetheless, this should not discourage the physician or the smoker patient from renewed quitting attempts. Most relapses are known to occur very early in the attempt to quit $[24,25]$. Therefore, a close follow-up in the early days after the quitting date (possibly within $72 \mathrm{~h}$ ) is important to handle relapse before it is too late. Nevertheless, there are subgroups of patients not showing the well-described steady decrease in the symptoms over time, but experiencing a return to the original severity late in the quitting attempt [12]. These patients are at the highest risk of relapse. Readministering a questionnaire to determine the progress of withdrawal symptoms severity at each visit may help to identify those patients experiencing a late elevation of symptoms.

Physicians should provide the patient with strategies designed to reinforce a commitment to stay tobacco-free, and assist patients who have relapsed [2]. Figure 1 illustrates an algorithm of reasonable tactics and recommendations for assisting smokers who have relapsed a few days to weeks after their quitting date.

Emphasizing the importance of continued efforts will help the patient to avoid feeling discouraged or frustrated by a relapse. The patient should be reminded that nicotine dependence is a chronic relapsing disease, and success may occur on later attempts. If a follow-up assessment shows that the patient is motivated, another quitting attempt should be encouraged as soon as possible by setting a new quitting smoking date [2].

The relapse can be used as a learning experience aimed at refining the cessation strategy with focused coping skills. It is important to elicit what led to the previous relapses, to identify the ways to prevent future relapse. A review of all possible smoking cueing/triggers can help to determine the cause. Was it stress, boredom, a social situation where everyone else was smoking? Ask about: negative affect and stress; being around other tobacco users, drinking alcohol, experiencing urges, smoking cues, and availability of cigarettes. Make an action plan outlining what steps to take next time a similar situation occurs to avoid smoking.

It may be necessary to increase the intensity of treatment and assistance. It is fundamental to individualize what to do next according to the symptoms and distress experienced by the patient:
- Increase the intensity and frequency of cessation counseling or refer the patient to a tobacco dependence specialist for a more intensive treatment if the patient is willing [2].

- Review/check timing/dosing of medications; the physician should review whether the patient used the medication in an effective manner and determine whether the medication was helpful.

- Address safety concerns. Recent studies of smokers and former smokers reveal that many smokers worry about using nicotine medications because of safety concerns [26, 27].

- Consider switching or adding varenicline or bupropion. In randomized controlled trials, varenicline is more effective than bupropion [28, 29]. All patients being treated with varenicline should be observed for neuropsychiatric symptoms including changes in behavior, hostility, agitation, depressed mood, and suicide-related events, including ideation, behavior, and attempted suicide. Combinations of smoking cessation medications appear to increase efficacy in smoking cessation compared to monotherapy [30]. Combinations that have been well-studied with proven benefit include the nicotine patch plus a more rapid release NRT such as gum, lozenge or spray, and bupropion plus NRT. The latter is approved for marketing as a combination therapy. Mechanisms underlying the effectiveness of combination NRT are thought to be a stable level of nicotine from the patch to relieve withdrawal symptoms plus the use of more rapid release preparations to deal with episodes of craving or other withdrawal symptoms. Combinations of other medications provide two different mechanisms for relief of withdrawal symptoms and/or antagonism of nicotine reinforcement from smoking relapses.

- Add a nicotine-free inhaler for those with a significant behavioral component of their tobacco dependence [31]. These products are plastic devices that are intended to provide a coping mechanisms for conditioned smoking cues by replacing some of the rituals associated with smoking gesture (e.g., hand-to-mouth action of smoking).

- Re-administer the questionnaire on withdrawal symptoms. During follow-up, compare responses to pre-quit responses.

- Increase frequency of follow-up, more frequent followup, particularly closer to quit date, make use of telephone or e-mail follow-ups [32].

- Review familial/social support; partner, household, friends, and colleagues support has a role to play in successful smoking cessation and smokers, who want to quit, should request their support. 


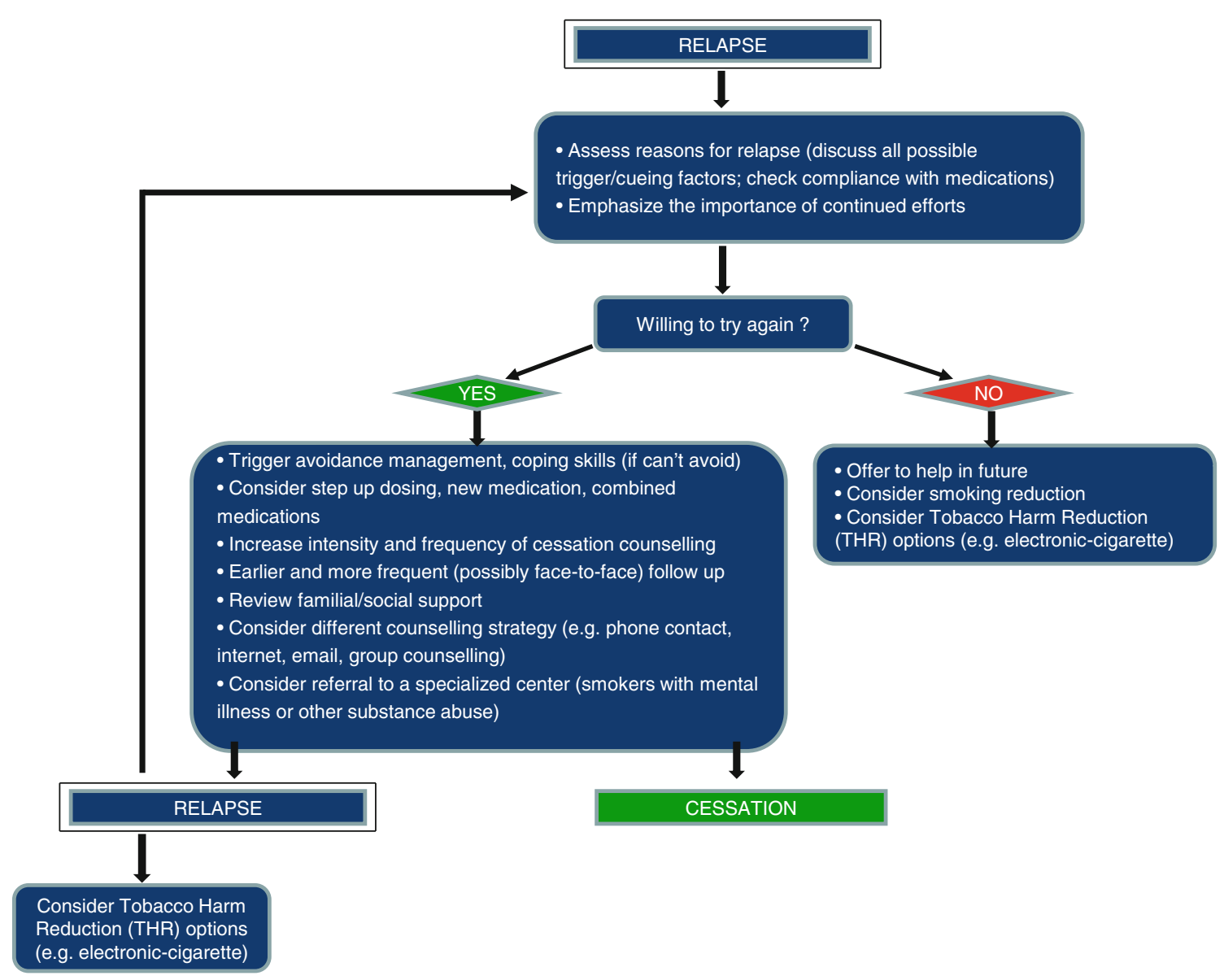

Fig. 1 Algorithm of possible strategies and recommendations for assisting relapsing smokers. The algorithm assumes a treated smoker attempting to quit smoking, who has a relapse a few days to weeks after a quitting date

Despite the emphasis on the importance of continued efforts, several patients may decline further assistance after relapse. Patients who have relapsed and are unwilling to try quitting again should receive a brief intervention designed to increase future quitting attempts [2]. For these smokers wait for a better moment, and then restart from zero considering the experience achieved from previous quitting attempts. As an alternative, smoking reduction may be a viable treatment approach to promote future smoking abstinence among smokers who are not ready to repeat a quitting attempt [33]. An additional option is to encourage the patient to switch to a less harmful source of nicotine. Studies of Swedish smokers who switch to a chewable form of smokeless tobacco (i.e., snuff) as a replacement for smoking show that the use of this product is associated with minimal health risks and reduced mortality from cardiovascular disease [34]. Recent research with electronic cigarettes, battery-operated products designed for the purpose of nicotine delivery has found them to be safe and effective in helping smokers remain abstinent [35-37].

\section{Conclusions}

Assisting smokers who have relapsed is not easy. The challenging path to successful smoking cessation runs via a constructive and supportive therapeutic alliance between the patient and a cessation specialist. The importance of forming this alliance stands in its potential to foster longterm treatment adherence and achieve optimal exploitation of individualized treatment plans.

However, realistic plans must also be taken into account that sustained cessation may not be achievable for many smokers, and alternative options should then be offered. Nicotine's pleasurable effects can be controlled, and the detrimental effects of the delivery mechanism can be attenuated by providing nicotine from less hazardous sources. Thus, advising smokers who cannot (or do not want to) quit to switch to either low-nitrosamine snuff or electronic cigarette could be an equally effective way to help smokers to become abstinent. This new emphasis on tobacco harm reduction as an exit strategy for smokers unable (or unwilling) to quit is a key paradigm shift in the 
management of relapse, which could save millions of lives world-wide.

Conflict of interest None of the authors have any competing interests to declare, with the exception of RP. RP has received lecture fees from Pfizer and GSK, a research grant from Pfizer, and he served as a consultant for Pfizer, Global Health Alliance for treatment of tobacco dependence, and Arbi Group Srl.

\section{References}

1. US Department of Health and Human Services (1990) The health benefits of smoking cessation (DHHS Publication No. (CDC) 90-8516). US Department of Health and Human Services, Public Health Service, Centers for Disease Control, Center for Chronic Disease Prevention and Health Promotion, Office on Smoking and Health, USA

2. Fiore MC, Jaen CR, Baker TB, Bailey WC, Benowitz N, Curry SJ, et al. (2008) Treating tobacco use and dependence: 2008 update. US Dept of Health and Human Services, Public Health Service.

3. Hughes J (2007) Effects of abstinence from tobacco: valid symptoms and time course. Nicotine Tob Res 9(3):315-327

4. Casella G, Caponnetto P, Polosa R (2010) Therapeutic advances in the treatment of nicotine addiction: present and Future. Ther Adv Chronic Dis 1(3):95-106

5. Tobacco Advisory Group of the Royal College of Physicians (2007) Harm reduction in nicotine addiction: helping people who can't quit. 978-1-86016-319-7

6. American Psychiatric Association (1994) Diagnostic and Statistical Manual of Mental Disorders, 4th edn. American Psychiatric Association, USA

7. World Health Organization (1992) The ICD-10 classification of mental and behavioural disorders: clinical descriptions and diagnostic guidelines. World Health Organization, Geneva

8. West R, Ussher M, Evans M, Rashid M (2006) Assessing DSMIV nicotine withdrawal symptoms: a comparison and evaluation of five different scales. Psychopharmacology (Berl) 184(3-4): 619-627 (Epub 2005 Nov 25)

9. Schnoll RA, Martinez E, Tatum KL, Glass M, Bernath A, Ferris D, Reynolds P (2011) Increased self-efficacy to quit and perceived control over withdrawal symptoms predict smoking cessation following nicotine dependence treatment. Addict Behav 36(1-2):144-147 (Epub 2010 Sep 24)

10. Hughes JR (2007) Effects of abstinence from tobacco: etiology, animal models, epidemiology, and significance: a subjective review. Nicotine Tob Res 9(3):329-339

11. Kenford SL, Fiore MC (2004) Promoting tobacco cessation and relapse prevention. Med Clin North Am 88(6):1553-1574 (review)

12. Piasecki TM, Fiore MC, Baker TB (1998) Profiles in discouragement: two studies of variability in the time course of smoking withdrawal symptoms. J Abnorm Psychol 107(2):238-251

13. Batra A (2011) Treatment of tobacco dependence. Dtsch Arztebl Int 108(33):555-564. doi:10.3238/arztebl.2011.0555 (Epub2011 Aug 19)

14. Partos TR, Borland R, Yong HH, Thrasher J, Hammond D (2012) Cigarette packet warning labels can prevent relapse: findings from the International Tobacco Control 4-Country policy evaluation cohort study. Tob Control (Epub ahead of print)

15. Devries KM, Kenward MG, Free CJ (2012) Preventing smoking relapse using text messages: analysis of data from the txt2stop trial. Nicotine Tob Res (Epub ahead of print)
16. Panlilio LV, Justinova Z, Mascia P, Pistis M, Luchicchi A, Lecca S, Barnes C, Redhi GH, Adair J, Heishman SJ, Yasar S, Aliczki M, Haller J, Goldberg SR (2012) Novel use of a lipid-lowering fibrate medication to prevent nicotine reward and relapse: preclinical findings. Neuropsychopharmacology. doi:10.1038/ npp.2012.31 (Epub ahead of print)

17. Tonstad S, Tonnesen $\mathrm{P}$, Hajek $\mathrm{P}$, Williams KE, Billing CE, Reeves KE (2006) Effect of maintenance therapy with varenicline for smoking cessation. JAMA 296:64-71

18. Agboola S, McNeill A, Coleman T, Leonardi Bee J (2010) A systematic review of the effectiveness of smoking relapse prevention interventions for abstinent smokers. Addiction 105(8): 1362-1380

19. Polosa R, Benowitz NL (2011) Treatment of nicotine addiction: present therapeutic options and pipeline developments. Trends Pharmacol Sci 32(5):281-289

20. Fahim RE, Kessler PD, Fuller SA, Kalnik MW (2011) Nicotine vaccines. CNS Neurol Disord Drug Targets 10(8):905-915

21. Caponnetto P, Polosa R (2008) Common predictors of smoking cessation in clinical practice. Respir Med 102:1182-1192

22. Castro Y, Costello TJ, Correa-Fernández V, Heppner WL, Reitzel LR, Cofta-Woerpel L, Mazas CA, Cinciripini PM, Wetter DW (2011) Differential effects of depression on smoking cessation in a diverse sample of smokers in treatment. Am J Prev Med 41(1):84-87

23. Sirey JA et al (2001) Stigma as a barrier to recovery: perceived stigma and patient-rated severity of illness as predictors of antidepressant drug adherence. Psychiatr Serv 52(12):1615-1620

24. Zhu S, Pierce JP (1995) A new scheduling method for time limited counseling. Prof Psychol Res Pract 26:624-625

25. Hughes JR, Keely J, Naud S (2004) Shape of the relapse curve and long-term abstinence among untreated smokers. Addiction 99:29-38

26. Carpenter MJ, Ford ME, Cartmell K, Alberg AJ (2011) Misperceptions of nicotine replacement therapy within racially and ethnically diverse smokers. J Natl Med Assoc 103(9-10):885894

27. Etter JF, Perneger TV (2001) Attitudes toward nicotine replacement therapy in smokers and ex-smokers in the general public. Clin Pharmacol Ther 69:175-183

28. Gonzales D, Rennard SI, Nides M, Oncken C, Azoulay S, Billing $\mathrm{CB}$ et al (2006) Varenicline, an a4b2 nicotinic acetylcholine receptor partial agonist, vs sustained-release bupropion and placebo for smoking cessation: a randomized controlled trial. JAMA 296:47-55

29. Jorenby DE, Hays JT, Rigotti NA, Azoulay S, Watsky EJ, Williams KE et al (2006) Efficacy of varenicline, an a4b2 nicotinic acetylcholine receptor partial agonist, vs placebo or sustained-release bupropion for smoking cessation: a randomized controlled trial. JAMA 296:56-63

30. Ebbert JO, Hays JT, Hurt RD (2010) Combination pharmacotherapy for stopping smoking: what advantages does it offer? Drugs 70(6):643-650

31. Caponnetto P, Cibella F, Mancuso S, Campagna D, Arcidiacono G, Polosa R (2011) Effect of a nicotine free inhalator as part of a smoking cessation program. Eur Respir J 38(5):1005-1011

32. Polosa R, Russo C, Di Maria A, Arcidiacono G, Morjaria JB, Piccillo G (2009) Feasibility of using e-mail counseling as part of a smoking-cessation program. Respir Care 54(8):1033-1039

33. Asfar T, Ebbert JO, Klesges RC, Relyea GE (2011) Do smoking reduction interventions promote cessation in smokers not ready to quit? Addict Behav 36(7):764-768 (Epub $2011 \mathrm{Feb} 12$ )

34. Roth D, Roth AB, Liu X (2005) Health risks of smoking compared with Swedish Snus. Inhal Toxicol 17:741-748

35. Polosa R, Caponnetto P, Morjaria JB, Papale G, Campagna D, Russo C (2011) Effect of an electronic nicotine delivery device 
(e-cigarette) on smoking reduction and cessation: a prospective 6-month pilot study. BMC Public Health 11(11):786

36. Caponnetto P, Polosa R, Auditore R, Russo C, Campagna D (2011) Smoking cessation with $e$-cigarettes in smokers with a documented history of depression and recurring relapses. Int $\mathbf{J}$ Clin Med 2:281-284
37. Caponnetto P, Polosa R, Russo C, Leotta C, Campagna D (2011) Successful smoking cessation with electronic cigarettes in smokers with a documented history of recurring relapses: a case series. J Med Case Rep 5(1):585 